\title{
One-step Generation of Phenotype-expressing Triple-knockout Mice with Heritable Mutated Alleles by the CRISPR/Cas9 System
}

\author{
Wataru FUJII'), Asuka ONUMA ${ }^{1)}$, Koji SUGIURA ${ }^{1)}$ and Kunihiko NAITO ${ }^{1)}$ \\ 1) Department of Animal Resource Sciences, Graduate School of Agricultural and Life Sciences, The University of Tokyo, \\ Tokyo 113-8657, Japan
}

\begin{abstract}
Triple-knockout mice generated by the one-step CRISPR/Cas9 system were examined for the effects of multiple gene modifications on each phenotype and individual gene function. Sixty embryos were transferred, and 9 pups were obtained; all 9 pups had mutations on 3 loci, and 7 pups showed mutations in all-alleles. F0 mice showed knockout phenotypes or no protein expression of target genes simultaneously, and these mutations were normally inherited in the next generation.
\end{abstract}

Key words: CRISPR/Cas9, Heritable, Mouse, Phenotype, Triple knockout

(J. Reprod. Dev. 60: 324-327, 2014)

$\mathbf{T}$ he CRISPR/Cas9 system consists of synthetic guide RNA (gRNA), which recognizes and binds with a target genome DNA, and Cas9 endonuclease, which is recruited to the target sequence by gRNA and induces DNA double-strand breaks and subsequent repair-associated mutations to a target sequence $[1,2]$. This system is expected to provide an easy, inexpensive and fast method for mammalian genome modification. Unlike the DNA binding domain-conjugated artificial endonucleases, such as zinc finger nucleases (ZFNs) and transcription activator-like effector nucleases (TALENs), the CRISPR/ Cas9 system is suitable for multiple genome mutations, because plural DNA-recognizing-molecules can be applied to multiple targets simultaneously at a constant concentration of Cas9 endonuclease. We previously developed a highly expressing Cas9 by addition of the 3' untranslated region (UTR) of mouse TATA box-binding protein-like 1 (Tbpl1) mRNA with a 95-bp polyadenine tail directly to the 3' UTR of Cas9 mRNA and reported efficient generation of mice with double mutations in the Hprt gene or a large-scale (more than $10 \mathrm{~kb}$ ) deletion between two Hprt target sites with almost no off-target effects [3]. To date, successful generations of Tet1 and Tet2 double-knockout mice, Tet1 and Tet2 biallelic rats with Tet3 monoallelic mutations and a quintuple-knockout immunodeficient mouse have reportedly been generated by a zygote-mediated CRISPR/ Cas9 system [4-6]. These studies focused most of their attention on the generation efficiency of the CRISPR/Cas9 system and chosen the target genes accordingly a similar function. Thus, to the best of our knowledge, the effects of multiple genome modifications on each gene phenotype have not yet been examined nor has there been any analysis of individual gene function.

Received: December 12, 2013

Accepted: March 26, 2014

Published online in J-STAGE: May 4, 2014

C2014 by the Society for Reproduction and Development

Correspondence: W Fujii (e-mail: awtrfj@mail.ecc.u-tokyo.ac.jp),

K Naito (e-mail: aknaito@mail.ecc.u-tokyo.ac.jp)

This is an open-access article distributed under the terms of the Creative Commons Attribution Non-Commercial No Derivatives (by-nc-nd) License $<$ http://creativecommons.org/licenses/by-nc-nd/3.0/>.
In the present study, we addressed whether triple-knockout mice generated by our CRISPR/Cas9 system expressed each phenotype or protein expression as reported in single-knockout mice. To that end, genes expressing easily observable phenotypes were selected for targets as follows. Tyrosinase (Tyr) is essential for melanin pigment biosynthesis, and a deficiency of it whitens the coat color of colored mouse strains [7]. Growth differentiation factor-8 (Gdf8), also known as myostatin, is an inhibitor of muscle development, and its deficiency induces a muscle hypertrophy phenotype [8]. We chose Hprt on the X chromosome for the third target because high-efficiency knockout of it was demonstrated in our previous study [3] and we expected to be able to easily detect its protein expression by immunoblotting. Human Hprt deficiency results in the hyperproduction of uric acid, known as Lesch-Nyhan syndrome, but no clear phenotype has been observed in mouse Hprt deficiency due to the presence of a compensation mechanism [9]. We tried to generate triple-knockout mice of these three genes using the CRISPR/Cas9 system with a highly expressing Cas9 we developed previously [3] and confirmed the expression of the same phenotypes as those reported in single-knockout mice of Tyr and Gdf8 and no expression of Hprt protein. Furthermore, we confirmed normal transmission of the modified genes to the next generation through multiple gene-deficient germline cells.

We synthesized three gRNAs targeting Tyr, Gdf8 and Hprt (Supplementary Table 1: on-line only), and these gRNAs and Cas9 mRNA were injected simultaneously into 60 fertilized eggs of C57BL/6N mice. Nine pups (15\%) were obtained, and their target sequences were confirmed by PCR-direct sequencing using tail tip DNAs. We found that all 9 pups had genome modifications in three target sites, and that 7 pups had modifications in all alleles, including biallelic mutations of three genes in females and biallelic mutations of Tyr and Gdf8 and hemiallelic mutation of Hprt in males (Fig 1A). We examined the off-target effects in 3 sites each for Tyr and Gdf 8 and detected no off-target effects in any of the examined sites (Supplementary Table 2: on-line only).

A white coat color was observed in all 9 pups, indicating the successful knockout of Tyr genes (Fig. 1B, C). Although 8 pups were completely white (Fig. 1B), one pup (\#8 in Supplementary Fig. 1: 
(A)

\begin{tabular}{cccccc}
\hline $\begin{array}{c}\text { No. of 2-cell embryos } \\
\text { transferred } \\
\text { (no. of recipients) }\end{array}$ & No. of pups & \multicolumn{2}{c}{$\begin{array}{c}\text { No. of mutated pups } \\
\text { (mutation of all alleles) }\end{array}$} & \multirow{2}{*}{$\begin{array}{c}\text { No. of pups with } \\
\text { mutation of all } \\
\text { alleles (\%) }\end{array}$} \\
\cline { 3 - 5 } & Tyr & Gdf8 & Hprt & \\
\hline $60(3)$ & 9 & $9(9)$ & $9(8)$ & $9(8)$ & $7(77.8)$ \\
\hline
\end{tabular}

(B)

(C)
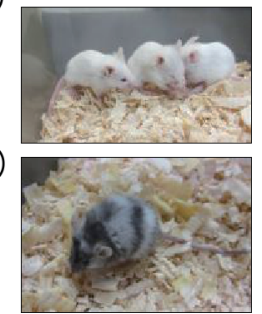

(D)

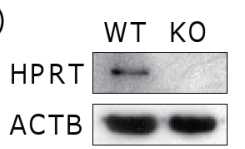

$(\mathrm{E})$
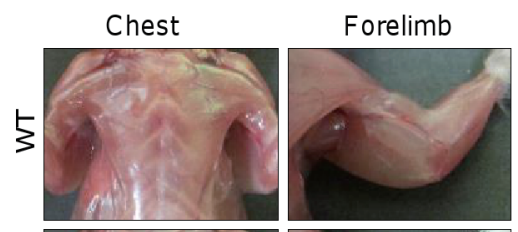

Hindlimb
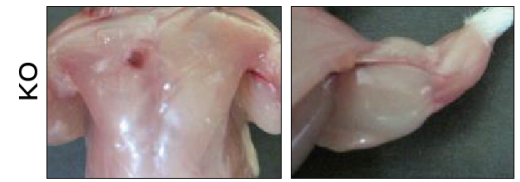
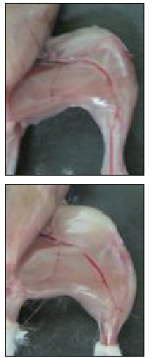

Fig. 1. Generation of triple-knockout mice and their phenotypes. Approximately $4 \mathrm{pl}$ of RNA solution containing $10 \mathrm{ng} / \mu \mathrm{l}$ each of Cas 9 mRNA, Tyr gRNA, Gdf8 gRNA and Hprt gRNA was injected into the cytoplasm of each one-cell embryo and cultured in M16 medium. Sixty 2-cell embryos were transferred into the oviductal ampullae (10 embryos per oviduct) of 3 recipients. (A) Generation efficiency of tripleknockout mice. (B) Three examples of the 8 pups (\#1-\#3 in Supplementary Fig. 1) with a white coat color over the whole body. (C) One of the 9 pups (\#8 in Supplementary Fig. 1) showed a black and white striped mosaic coat pattern. (D) Immunoblotting of Hprt and beta-Actin using a muscle sample from an Hprt biallelic knockout female pup (\#1 in Supplementary Fig. 1). (E) Hyperproduction of skeletal muscle in a knockout mouse (KO, \#1 in Supplementary Fig. 1) compared with a wild-type mouse (WT).

(A)

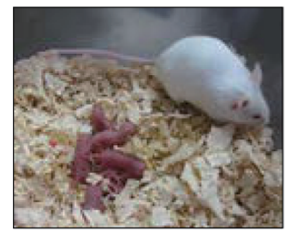

(B)

Tyr

AAGGGGTGGATGACCGTGAGTCCTGGCCC WT AAGGGGTGGATGACCG---- cTCCTGGCC -4, +1 AAGGGGTGGATGACCGTGAGgTCCTGGCC +1

\section{Gdf8}

AGCCCAACTGTGGATATATCTCAGACCCGTCA WT AGCCCAACETGTGGATATATCTCAGACCCGTCA +1 AGCCCAAC----CA -22

\section{Hprt}

GGTCCCGTCATGCCGACCCGCAGTCCCAGC WT

GGTCCC-------- $A G C-21$

GGTCCC------GACCCGCAGTCCCAGC -8
(C)

F1
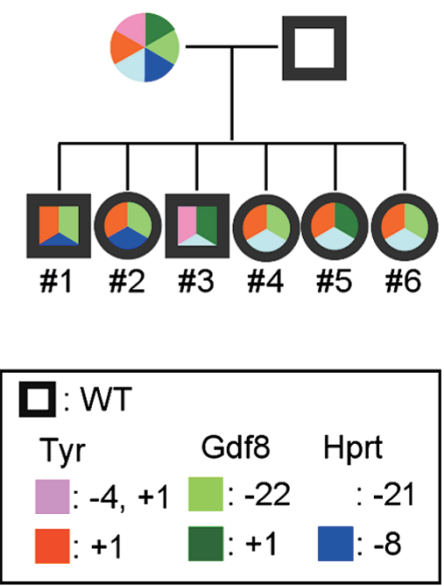

Fig. 2. Inheritance of multiple mutated alleles in the next generation. (A) Six F1 pups were obtained from a triple-knockout F0 female (\#2 in Supplementary Fig. 1) mated with a wild-type male. (B) Target sequences of both alleles of the F0 female were confirmed by PCR-direct sequencing using tail tip DNAs and are shown here with wild-type sequences. (C) A pedigree obtained from sequence analyses of the F0 female and F1 pups. 
on-line only) had white and black stripes indicating mosaicism (Fig. 1C). Hprt protein was not detected by immunoblotting using muscle samples from an Hprt biallelic knockout pup (\#1 in Supplementary Fig. 1), indicating inhibition of gene expression by biallelic destruction (Fig. 1D). A muscle hypertrophy phenotype was observed by external appearance in 8 pups with biallelic knockout of $G d f 8$ (data not shown). Clear hyperproduction was observed in the subcutaneous muscle phenotype compared with wild-type mice, indicating the successful knockout of $G d f 8$ genes (Fig. 1E). These results demonstrate that the CRISPR/Cas9 system is able to modify 3 target sites ( 6 alleles in females and 5 alleles in males) simultaneously and efficiently, and that the phenotypes of triple-knockout mice are comparable to those of corresponding single-knockout mice [7-9], suggesting the possibility of generating mice with multiple knockout mutations by the CRISPR/Cas9 system for gene function analysis.

The targeted sequences of a female mouse (\#2 in Supplementary Fig. 1) with biallelic mutation in 3 genes are shown in Fig. 2B, and 6 F1 pups were obtained from this female mouse after crossing with a wild-type male mouse (Fig 2C). We examined the target sequences of these $\mathrm{F} 1$ pups and found that 3 sites of each allele were identical to one of the mutated alleles of the mother in all pups (Fig. 2C and Supplementary Fig. 2: on-line only). These results indicate that the female mouse in question was certainly a mouse with targeted mutations in all alleles including germline cells and that the modified genes were transmitted normally to the next generation through multiple gene-deficient germline cells.

In the present study, all target alleles of almost all the obtained pups were mutated. A previous report concerning multi-gene targeting by CRISPR/Cas showed lower mutation efficiencies and suggested the necessity of designing plural gRNAs for each gene in order to improve the gene destruction efficiency [6]. The Cas9 mRNA used in the present study is expected to be translated stably, because the construct contains a partial sequence of the Tbpl1 3' UTR with a 95-base polyadenine [3], which has been reported to induce high translation efficiency [10]. In addition, the present target sequences had more than $40 \% \mathrm{GC}$ contents, according to the previous indication that the high GC contents of target sequence might be correlated to the high genome modification efficiencies [3]. These optimizations might be attributed to the high genome-modification efficiency in the present study, and it should be possible to adopt the present system for destruction of more genome loci simultaneously. On the other hand, the off-target effects may increase along with the increasing number of target loci. Recently, we reported the application of an offset-nicking method, which consisted of a Cas9-nickase and paired gRNAs, to mouse zygotes directly and showed that the method was suitable for generation of genome-modified mice and avoiding off-target effects [11]. By applying this method, mice with multiple knockout mutations can be efficiently generated without off-target risks.

A white coat color, the Tyr knockout phenotype, was observed in all 9 pups in spite of the in-frame mutation in four pups (\#4, \#5, $\# 7$ and \#8 in Supplementary Fig. 1). Their Tyr proteins should be expressed with only few amino acid mutations, suggesting that the mutated amino acids were essential for the tyrosinase activity and that the present Tyr target locus is critical for the physiological function. Regarding Hprt, 8 pups showed mutations in all alleles, but no clear phenotype was observed, as reported previously [9]. Although we examined the Hprt protein expression in only 1 pup (\#2 in Supplementary Fig. 1), the translation initiation sites were removed, and therefore Hprt protein should not be expressed in all but one of the pups, which escaped removal of the translation initiation site and showed an in-flame mutation (\#3 in Supplementary Fig. 1). Muscle hypertrophy, the $G d f 8$ knockout phenotype, was observed in the 8 pups showing mutations in all alleles including 2 pups showing an in-flame mutation (\#4 and \#8 in Supplementary Fig. 1). This should indicate the importance of mutated amino acids for myostatin function. On the other hand, this pup had mosaicism as shown by its coat color, and there is a possibility that the mutation pattern of the tail tip DNA was different from that of the target tissues. Although almost all analyses of genome-modified animals to date have been performed using tail tip DNA obtained from F0 mice, the present results of Tyr gene modification and our previous report [3] indicate the risk of mosaicism in genome modification using the CRISPR/Cas9 system, and therefore tail tip DNA of F0 mice does not always reflect the strict genotype. We propose the use of F1 mice for systematic analysis of phenotypes in knockout mice generated by the CRISPR/Cas9 system.

In the present study, almost all of the obtained pups showed mutations in all target alleles, and the mutated alleles were inherited simultaneously in the next generation. Mutated Tyr and $G d f 8$ expressed phenotypes as reported in single-knockout mice. These results indicate that a zygote-mediated CRISPR/Cas9 system is usable for efficient analysis of plural gene functions in vivo. This system is expected to contribute to studies on the complicated features of the in vivo functions of plural genes, especially family genes or regulons that have compensation functions.

\section{Methods}

\section{Ethics Statement}

All animal care and experiments conformed to the Guidelines for Animal Experiments of The University of Tokyo and were approved by the Animal Research Committee of The University of Tokyo.

\section{Construction of $g R N A$ coding vectors}

Target sequences for Tyr and Gdf8 (Supplementary Table 1) were designed, and plasmid vectors coding gRNAs with T3 promoter for each target were synthesized according to a previous study [3]. Regarding Hprt, previously reported target sequence (Supplementary Table 1) and the gRNA-coding vector [3] were used in the present study. These vectors were sequenced using a commercial sequencing kit (Applied Biosystems, Foster City, CA, USA) and a DNA sequencer (Applied Biosystems) according to the manufacturer's instructions.

\section{In vitro transcription of CAS9, gRNAs and ZFNs}

For in vitro synthesis of CAS9 mRNAs, CAS9-coding vector reported in a previous study [3] was linearized by SphI and transcribed in vitro with T3 RNA polymerase (Promega) in the presence of m7G(5')ppp(5')G to synthesize capped RNA. In the case of gRNAs, each gRNA vector was linearized by DraI and transcribed by the same procedure but without $\mathrm{m} 7 \mathrm{G}\left(5^{\prime}\right) \mathrm{ppp}\left(5^{\prime}\right) \mathrm{G}$ to avoid formation of the cap structure. The RNA transcripts were precipitated with 
absolute ethanol, washed and resuspended in RNase-free water. The RNA solutions were stored at $-80 \mathrm{C}$ until use.

\section{Microinjection}

Sexually immature female C57BL/6NCr mice (4 weeks old) were superovulated by intraperitoneal injection of $7.5 \mathrm{IU}$ eCG followed by $7.5 \mathrm{IU}$ hCG at an interval of $48 \mathrm{~h}$ and mated overnight with $\mathrm{C} 57 \mathrm{BL} / 6 \mathrm{NCr}$ male mice that were $>12$ weeks old. Zygotes were collected after $20 \mathrm{~h}$ of hCG injection by oviductal flushing, and zygotes with pronuclei were put into M2 medium. Microinjection was performed using a microscope equipped with a microinjector (Narishige). Approximately 4 pl of RNA solution containing $10 \mathrm{ng} /$ $\mu 1$ each of CAS9 mRNA, Tyr gRNA, Gdf8 gRNA and Hprt gRNA was injected into the cytoplasm of each zygote using continuous pneumatic pressure. After injection, all zygotes were cultured in M16 medium and subjected to the following experiments.

\section{Embryo transfer and mutation analysis of obtained pups}

Two-cell embryos injected with CAS9 mRNA and gRNAs were transferred into the oviductal ampullae (10 embryos per oviduct) of 7-8-week-old female ICR mice mated with vasectomized ICR males at the previous night. After birth, genome DNA was extracted from the tail tips and subjected to PCR for on-target (Supplementary Table 1) and off-target loci (Supplementary Table 2) using the primers shown in Supplementary Table 3. The off-target loci for $T y r$ and $G d f 8$ target sequences, which were newly designed in the present study, were searched using TagScan [12]. Off-target effect for Hprt was not analyzed in the present study, as a previous study showed no off-target effect in five off-target loci for Hprt [3]. PCR products were purified by agarose gel electrophoresis, and the extracted fragments were directly sequenced as described earlier in the text. The predicted mutation pattern of each allele was analyzed by sequence data according to a previous protocol [3].

\section{Immunoblotting of muscle-derived protein}

Western blot analysis was performed according to the standard protocol. Five-millimeter pieces of muscle that were obtained from the legs of knockout and wildtype mice were homogenized with a Potter homogenizer and were suspended in Laemmli buffer. The antibodies used were anti-HPRT polyclonal antibody (sc-20904, Santa
Cruz Biotech, Santa Cruz, CA, USA) and anti- $\beta$-actin polyclonal antibody (GTX109639, GeneTex, Irvune, CA, USA). To visualize the protein-bound antibodies, horseradish peroxidase-conjugated anti-goat IgG and anti-rabbit IgG (Jackson ImmunoResearch Laboratories, West Grove, PA, USA) were used for the second layer, respectively, followed by a detection procedure using an ECL detection kit (Amersham-Pharmacia) according to the manufacturer's protocol.

\section{Acknowledgments}

We thank Mr. K. Nakamura for technical advice. This study was supported by a Grant-in-Aid for Scientific Research (25892010 to WF and 20188858 to KN) from the Japan Society for the Promotion of Science.

\section{References}

1. Cong L, Ran FA, Cox D, Lin S, Barretto R, Habib N, Hsu PD, Wu X, Jiang W, Marraffini LA, Zhang F. Multiplex genome engineering using CRISPR/Cas systems. Science 2013; 339: 819-823.

2. Mali P, Yang L, Esvelt KM, Aach J, Guell M, DiCarlo JE, Norville JE, Church GM. RNA-guided human genome engineering via Cas9. Science 2013; 339: 823-826.

3. Fujii W, Kawasaki K, Sugiura K, Naito K. Efficient generation of large-scale genomemodified mice using gRNA and CAS9 endonuclease. Nucleic Acids Res 2013; 41: e187.

4. Wang H, Yang H, Shivalila CS, Dawlaty MM, Cheng AW, Zhang F, Jaenisch R. Onestep generation of mice carrying mutations in multiple genes by CRISPR/Cas-mediated genome engineering. Cell 2013; 153: 910-918.

5. Li W, Teng F, Li T, Zhou Q. Simultaneous generation and germline transmission of multiple gene mutations in rat using CRISPR-Cas systems. Nat Biotechnol 2013; 31: 684-686.

6. Zhou J, Shen B, Zhang W, Wang J, Yang J, Chen L, Zhang N, Zhu K, Xu J, Hu B, Leng Q, Huang X. One-step generation of different immunodeficient mice with multiple gene modifications by CRISPR/Cas9 mediated genome engineering. Int J Biochem Cell Biol 2014; 46: 49-55.

7. Le Fur N, Kelsall SR, Mintz B. Base substitution at different alternative splice donor sites of the tyrosinase gene in murine albinism. Genomics 1996; 37: 245-248.

8. McPherron AC, Lawler AM, Lee SJ. Regulation of skeletal muscle mass in mice by a new TGF-beta superfamily member. Nature 1997; 387: 83-90.

9. Wu CL, Melton DW. Production of a model for Lesch-Nyhan syndrome in hypoxanthine phosphoribosyltransferase-deficient mice. Nat Genet 1993; 3: 235-240.

10. Yamagata K, Yamazaki T, Yamashita M, Hara Y, Ogonuki N, Ogura A. Noninvasive visualization of molecular events in the mammalian zygote. Genesis 2005; 43: 71-79.

11. Fujii W, Onuma A, Sugiura K, Naito K. Efficient generation of genome-modified mice via offset-nicking by CRISPR/Cas system. Biochem Biophys Res Commun 2014; 445 791-794.

12. Iseli C, Ambrosini G, Bucher P, Jongeneel CV. Indexing strategies for rapid searches of short words in genome sequences. PLOS ONE 2007; 2: e579. 